\title{
BIOÉTICA CLÍNICA COMO PARTE INTEGRANTE DE UMA MEDICINA DOS VALORES E AFETOS
}

\author{
CLINICAL BIOETHICS AS AN INTEGRAL PART OF A MEDICINE OF \\ VALUES AND AFFECTIONS
}

\section{LA BIOÉTICA CLÍNICA COMO PARTE INTEGRAL DE UNA MEDICINA DE VALORES Y AFECTOS}

\section{DOI: $10.22481 /$ rbba.v10i02.9895}

Fernanda Borges Keid Comissões de Dir. Médico e da Advocacia na Mediação e Conciliação da OAB/SP, São Paulo, Brasil Lattes: http://lattes.cnpq.br/6417386983266816 Endereço eletrônico: fernandakeid@ @otmail.com

\begin{abstract}
RESUMO
Da assimetria relacional entre médicos e pacientes, emergem valores humanos morais capazes de impactar negativamente essa interação, dificultando a comunicação e a tomada de decisões. Em situações de vulnerabilidade, esses conflitos morais tornam-se agravados. Métodos deliberativos em Bioética surgem como instrumento na condução de dilemas morais. Este artigo se trata de uma revisão bibliográfica, cujo objetivo é demonstrar como a medicina pode se tornar menos defensiva, diminuindo as distâncias entre médico e paciente, por intermédio da Bioética. Fica revelado que o cuidado com a dor e com o sofrimento humano é o grande desafio a ser trabalhado pela área da saúde e, assim, deve ser constante a busca de um olhar mais aprofundado, capaz de perceber cada indivíduo na sua unidade, constituída de corpo e alma. A evolução tecnológica aumentou as distâncias entre seres humanos e, com isso, a Bioética Principialista precisou evoluir. Embora as deliberações bioéticas não sejam as soluções definitivas
\end{abstract}


para os dilemas morais, elas podem trilhar caminhos para uma medicina mais humanizada e mais afetuosa.

Palavras-chave: Bioética. Conflito. Deliberação. Humanização.

\title{
RESUMEN
}

De la asimetría relacional entre médicos y pacientes surgen valores morales humanos capaces de impactar negativamente esta interacción, dificultando la comunicación y la toma de decisiones. En situaciones de vulnerabilidad, estos conflictos morales se agravan. Los métodos deliberativos en bioética surgen como un instrumento en la conducción de los dilemas morales. Este artículo se trata de una revisión bibliográfica, cuyo objetivo es demostrar cómo la medicina puede volverse menos defensiva, reduciendo la distancia entre médico y paciente, a través de la Bioética. Se revela que el cuidado con el dolor y sufrimiento humano es el gran desafío a ser abordado por el área de salud, por lo que la búsqueda de una mirada más profunda debe ser constante, capaz de percibir a cada individuo en su unidad, compuesta de cuerpo y alma. La evolución tecnológica aumentó las distancias entre los seres humanos y, con ello, la Bioética Principialista necesitaba evolucionar. Si bien las deliberaciones bioéticas no son la solución definitiva a los dilemas morales, pueden allanar el camino para una medicina más humana y afectiva.

Palabras clave: Bioética. Conflicto. Deliberación. Humanización.

\begin{abstract}
From the relational asymmetry between physicians and patients, human moral values emerge capable of negatively impacting this interaction, making communication and decision-making difficult. In situations of vulnerability, these moral conflicts become aggravated. Deliberative methods in Bioethics emerge as an instrument in dealing with moral dilemmas. This article is a bibliographical review, whose objective is to demonstrate how medicine can be made less defensive, reducing the distances between doctor and patient, through Bioethics. It is revealed that the care of pain and human suffering is the great challenge to be worked on by the health area and, therefore, the search for a deeper look must be constant, capable of perceiving each individual in their unit, consisting of body and soul. Technological evolution increased the distances between
\end{abstract}


human beings and, with that, Principialist Bioethics needed to evolve. Although bioethical deliberations are not the definitive solutions to moral dilemmas, they can pave the way for a more humane and more affectionate medicine.

Keywords: Bioethics. Conflicts. Deliberations. Humanization.

\section{INTRODUÇÃO}

Pensar a Bioética pressupõe conhecer os valores humanos e como as suas divergências impactam as relações humanas. Esses valores ficam proeminentemente sobressaltados em situações de vulnerabilidade, como aquelas ocasionadas em âmbito hospitalar, em que o limiar entre padecimento e cura, vida e morte, saúde e doença levam os indivíduos à real condição de seres humanos hipossuficientes e finitos. Pensar a Bioética é perceber sua intersecção com os direitos humanos, na medida em que ambos compartilham o princípio da dignidade da pessoa humana e os bens básicos e invioláveis relacionados à medicina, às ciências da vida e às tecnologias a elas associadas (OLIVEIRA, 2011). Pensar a Bioética é, portanto, parte fundamental de uma medicina mais humanizada, mais caridosa, menos patriarcal, mais próxima do indivíduo vulnerado pela doença.

Da relação médico-paciente nascem conflitos e dilemas de valores, notadamente em face das emoções que permeiam as situações limítrofes, em especial no fỉm de vida. A autonomia, portanto, há de ser pensada sob o aspecto da vulnerabilidade e não da capacidade jurídica individual.

A consciência bioética tem, nesse sentido, como campo de atuação as vulnerabilidades humanas, sendo imprescindível o diálogo entre as diferentes perspectivas pessoais dos atores envolvidos (VASCONCELOS, 2020).

Da milenar medicina paternalista, baseada unicamente na experiência e no poder do conhecimento individual do médico, caminha-se à medicina baseada em evidências, que oferece dados objetivos e concretos, bem informados, para que o paciente possa ser capaz de tomar suas próprias decisões, em respeito à sua autonomia (URBAN, 2003).

Diante dessas perspectivas, o presente artigo consiste em uma revisão bibliográfica de literatura daquilo que já vem sendo publicado sobre o assunto nos últimos anos. A pesquisa foi realizada nas bases de dados da Biblioteca Virtual de Saúde - BVS. Utilizou-se também obras

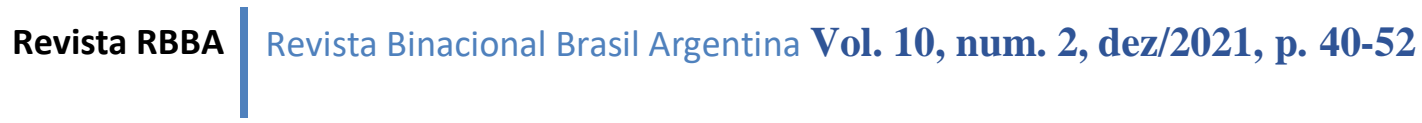


que abordam, além dos princípios bioéticos, importantes nuances da relação médico-paciente e seu contexto fomentador de dilemas ético-morais.

A pretensão é, deste modo, avaliar como o uso de metodologias deliberativas de valores em conflito, associadas com uma comunicação humanizada nas relações em saúde, podem contribuir para uma ciência médica mais afetuosa.

\section{A RELAÇÃO MÉDICO-PACIENTE}

A relação médico-paciente há tempos é considerada assimétrica, tanto diante do conhecimento técnico-científico portado pelo profissional, quanto do próprio domínio das circunstâncias vivenciadas. De acordo com Vasconcelos (2020), essa assimetria pode ser constatada no decurso de tempo, em face dos poderes médicos diversos, que se sobrepunham ao desconhecimento técnico dos pacientes acerca da medicina, como também em vista do poder mágico ou cognitivo ou tecnológico ou científico, a depender da época histórica.

Profissionais de saúde, detentores de conhecimento técnico-científico, com frequência, distanciam-se de seus pacientes, sendo imprescindível a manutenção do diálogo constante, com o repasse de informações claras e a confirmação de que os fatos transmitidos foram adequadamente compreendidos. Adicionalmente, a disparidade no nível de equilíbrio emocional dos envolvidos também precisa ser (re)considerada, na medida em que pacientes e familiares, além de precisarem lidar com suas próprias angústias, necessitam tomar decisões difíceis acerca das próprias vidas (CARREIRO, 2011).

Mesmo àquele paciente autônomo, que se revela aparentemente capaz e consciente para a tomada de decisões, ao se deparar com sua vulnerabilidade, em face da sua própria condição de saúde, ratifica a relação de dominação. Nesse sentido, considerando o enfoque da relação intersubjetiva entre médicos e pacientes, faz-se necessário pensar nessa assimetria, de modo a aprofundar a compreensão, portanto, de conceitos que demonstram a efetiva hierarquização dos discursos e o dificultoso diálogo simétrico entre médicos e pacientes, potencializadores de conflitos. (VASCONCELOS, 2020).

Ainda, o adoecimento pode levar o paciente à sensação de perda do controle sobre seu próprio corpo, emergindo sensações de impotência e frustração, o que enseja a necessidade de informações precisas sobre seu diagnóstico, prognóstico e tratamento, além de suporte emocional. Cabe ao profissional estabelecer o elo de confiança e empatia, para que a Revista RBBA $\mid$ Revista Binacional Brasil Argentina Vol. 10, num. 2, dez/2021, p. 40-52 
comunicação seja mais humanizada possível, de modo a superar modelos que reforcem relações de poder e hierarquia (PAES, ESCH, 2021).

Demais disso, imprescindível mencionar a vulnerabilidade do profissional de saúde, acarretada pelas intercorrências ou por eventos adversos, que independem de sua capacidade técnica. Essas também são as verdades de médico. O reconhecimento dessas vulnerabilidades diminui a assimetria existente entre médico-paciente, aumentando a capacidade de ser empático. E, ainda, consoante alertado por Meira (2004, p.250):

Muitas vezes o médico procura em prazo pequeno atender um número maior de clientes, outras vezes em razão da falta de tempo ou de organização esquece algum paciente por longo tempo. Dependente de múltiplas atividades que pretende exercer ao mesmo tempo, não tem a preocupação com o horário estabelecido. Coloca-se nesses momentos acima do bem e do mal entendendo que ao paciente só resta esperar. Essa espera é agonizante. Agonia que passa a irritação, quando por razões várias, seja de amizade ou de compromissos financeiros, o médico discrimina na escolha de quem primeiro deve ser atendido. O paciente não vai à procura do médico por vontade própria, sequer para satisfazer um desejo, vai porque tem uma necessidade premente de socorro. Nessa ânsia pelo socorro e nessa espera pelo atendimento é que acontece o contato das duas partes. A solidariedade social da espera promulgada em gabinetes políticos, também, é evidenciada nos consultórios e ambulatórios médicos de todos os níveis.

Desse cenário, surgiu a Recomendação do CFM n. ${ }^{\circ}$ 1/2016, que dispõe sobre o processo de obtenção do consentimento livre e esclarecido na assistência médica, sendo, portanto, importante marco normativo sobre a comunicação entre os atores dessa relação (BRASIL, 2016).

Para uma relação saudável, é essencial que a comunicação e a confiança sejam elementos presentes, de modo a haver mitigação das vulnerabilidades e da assimetria preexistentes. Ademais, o empoderamento do paciente enfermo permite uma tomada de decisões de forma mais segura, confiante e autônoma, em atendimento ao próprio fundamento constitucional da dignidade da pessoa humana (PAZINATTO, 2011).

Nesse mesmo sentido, aduz Rocha et al (2011, p.2):

A construção da relação médico-paciente nasce da abordagem utilizada pelo profissional, devendo imprimir características humanas, subjetivas, de uma forma natural. Não deve se limitar à objetividade das tecnologias. Deve também usar de sua anamnese para que, através das queixas relatadas pelo

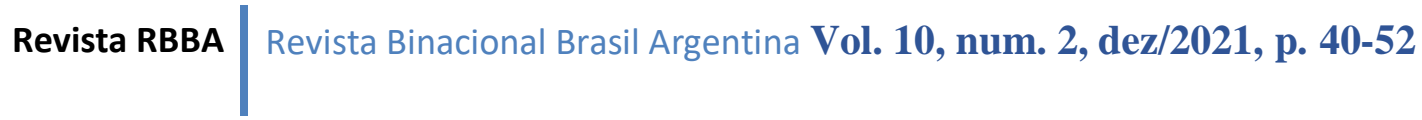


doente e também procurando conhecer o estilo de vida do indivíduo, desenvolva o tratamento mais eficaz. Já que a relação é um aspecto chave para a melhoria da qualidade do serviço, deve-se buscar a personalização da assistência humanizada do atendimento e o direito à informação, assim como o consentimento informado.

Portanto, deve-se reconhecer que a relação médico-paciente guarda grandes desafios na sua construção, que demanda esforços de ambas as partes, porquanto dessa relação pode ocorrer a potencialização de conflitos de valores morais, quando a comunicação e a empatia não se mostram presentes.

Segundo Souza et al (2012, p.185):

Na bioética da relação médico-paciente está o conflito entre o emocional e o racional; o maior desgaste do profissional médico não deve ao número de horas trabalhadas, mas à intensidade emocional com que a vivência de todos os seus atos, pois eles significam tratar com a vida, a honra e a saúde de outras pessoas. Contudo, na maioria das vezes este conflito é desconhecido tanto pelo médico quanto pela sociedade.

Uma relação médico-paciente satisfatória só se mostra possível quando ambos estão dispostos a isso, levando-se em consideração aspectos mentais e emocionais subjacentes aos próprios comportamentos que ficam escancarados diante da vulnerabilidade do binômio saúdedoença, repercutindo, pois, em uma gama de fenômenos emergentes, os quais poderão ser solucionados por intermédio da Bioética. (NASCIMENTO JUNIOR, GUIMARÃES, 2009).

\section{A BIOÉTICA}

Não se pode olvidar que a Bioética é fruto do julgamento de Nuremberg, ocasião em que ficaram comprovados que experimentos com seres humanos, em nome da descoberta de novos avanços científicos, foram realizados por médicos com pessoas detidas nos campos de concentração, independentemente de seus consentimentos (CARREIRO, 2011).

Consoante Carreiro (2011), muito embora o vocábulo "bioética" ainda não existisse à época, o julgamento dos médicos nazistas e os princípios que deles decorreram, com o estabelecimento de parâmetros éticos para pesquisas envolvendo seres humanos, pronunciaram uma nova era em que a eticidade do desenvolvimento técnico-científico ganhou relevância, assim como os valores morais em torno da relação médico-paciente.

Revista RBBA Revista Binacional Brasil Argentina Vol. 10, num. 2, dez/2021, p. 40-52 
O termo bioética, derivado das palavras bios (vida) e ethike (ética), foi adotado pela primeira vez no início da década de 70, por Van Rensselaer Potter, podendo ser definido como um estudo sistemático de dimensões morais - incluindo visão, decisão, conduta e normas morais - das ciências da vida e do cuidado com a saúde, utilizando-se, para tanto, de uma série de metodologias éticas em um contexto multidisciplinar (PESSINI, BARCHIFONTAINE, 2005).

Além de Potter, ganha relevância as ideias surgidas com Andre Hellenger, obstetra holândes, que introduziu o termo Bioética pela primeira vez na academia, com maior enfoque nos desafios enfrentados pela medicina com o desenvolvimento tecnológico, com a escassez de recursos e com a necessidade de conexão com questões populacionais (CARRERO, 2011).

Convém destacar que a Bioética Potteriana, para Carreiro (2011), diz respeito à reflexão ética aplicada a temas ecológicos, ao passo que a aquela defendida por Andre Hellegers é mais centrada na medicina e suas interfaces, centralizando-se como uma Bioética Biomédica, posteriormente denominada Bioética Clínica.

O auge da Bioética mundial, de essência unicamente teórica, se deu com a publicação do livro de Beauchamp e Childress em 1979, quando foram sistematizados os quatro princípios da ética médica, quais sejam: autonomia, beneficiência, não-maleficiência e justiça, os quais denominaram a Teoria Principialista (BEAUCHAMP, CHILDRESS, 2002).

Significa dizer que a Bioética se expõe não mais apenas como um pensamento isolado, mas como uma forma institucionalizada, passando a contar com espaço próprio de atuação, em defesa do ser humano individualmente considerado. A Bioética surge, portanto, em defesa do homem e da sua sobrevivência, com melhoria na qualidade de vida, trazendo a ideia de homem holístico e revelando-se uma crítica aberta ao positivismo (URBAN, 2003).

Contudo, é possível concluir que a aplicação unicamente da Teoria Principialista depende de expectativas, objetivos e julgamentos de valores envolvidos em dada situação médica, sendo muito provável que sejam diferentes a cada um dos participantes da relação médico-paciente, que por certo envolve membros da equipe médico-hospitalar, familiares e o próprio paciente.

De acordo com Urban (2003), no Brasil, a Bioética surgiu tardiamente na segunda metade dos anos 80, o que repercutiu num atraso para a evolução dos profissionais de saúde "bioeticamente" capacitados a intervir de maneira hábil e direta nos dilemas morais surgidos em ambientes sensíveis, como os hospitalares.

Revista RBBA $\mid$ Revista Binacional Brasil Argentina Vol. 10, num. 2, dez/2021, p. 40-52 
Já em 2005, com a elaboração da Declaração Universal sobre Bioética e Direitos Humanos (DUBDH), houve a reunião de quinze princípios aplicáveis, tanto na medicina quanto nas pesquisas científicas, sempre tendo como alvo a preservação da dignidade da vida humana, passando a ser, dessa forma, mais um balizador das relações médico-paciente e das vulnerabilidades decorrentes desse entrosamento (PAZINATTO, 2019).

A Bioética encontra-se, assim, em um espaço multidisciplinar, no qual há imposição de reflexões acerca das teorias éticas e das questões concernentes às ciências da vida, à medicina e às tecnologias associadas, o que exige intervenções com a participação ativa dos mais diversos profissionais da saúde, do Direito, filósofos, teólogos, sociólogos, antropólogos, para que todas as perspectivas valorativas sejam consideradas em um dilema (CARREIRO, 2011).

\section{A BIOÉTICA CLÍNICA: UMA INTERVENÇÃO NECESSÁRIA}

Diante de uma sociedade globalizada e pluralista, o imenso desenvolvimento biotecnológico, a instrumentalização da morte nos hospitais e a influência de aspectos Econômicos e legais sobre a tomada de decisão médica fez com que surgisse um novo campo da ciência, que visa fortalecer a aliança entre o conhecimento científico e humanístico, intervindo em situações limítrofes como fim de vida, reprodução humana, pesquisas em seres humanos, além da aplicação de recursos cada vez mais escassos em saúde (URBAN, 2003).

O termo Bioética Clínica, como já mencionado, surgiu com Andre Hellegers e está intimamente relacionado às decisões éticas na prática médica, a partir dos princípios basilares bioéticos - autonomia, beneficiência, não-maleficiência e justiça (FIGUEIREDO, 2011). O autor destaca:

(...) o bioeticista espanhol Diego Gracia faz duas observações: 1) adverte que na ética médica não se deve começar estabelecendo grandes princípios, mas sim por estudos dos casos concretos, tendo, por consequência, que toda tomada de decisão tem como ponto de partida a história clínica; 2) considera que a ética clínica não pode ser entendida como mera aplicação dos princípios estabelecidos pela bioética fundamental. Assim, Gracia pondera que a bioética torna-se disciplina médica à medida que analisa as dimensões morais do julgamento clínico.” (FIGUEIREDO, 2011, p. 347)

Para Urban (2003), a Bioética Clínica dificulta o trabalho individual, pois a interdisciplinaridade torna-se mandamental, na medida em que há necessidade de intervenções

Revista RBBA $\mid$ Revista Binacional Brasil Argentina Vol. 10, num. 2, dez/2021, p. 40-52 
de muitas áreas de conhecimento que envolvem esse paciente holístico, permeado de valores pessoais e crenças.

Nas palavras de Carreiro (2011, p.48):

Assim, a Bioética que se preocupa com o valor que se concretiza na possibilidade de cada indivíduo escolher o seu próprio destino, contém no seu cerne essa incongruência fundamental por possuir fórmulas prontas para a resolução de todos os conflitos bioéticos, e essa incongruência permanecerá presente enquanto não for aceita a proposta de mediação dos conflitos bioéticos com base num espírito não-normativo, não-imperativo e pautada pelo reconhecimento da diferença moral da humanidade.

Da relação médico-paciente, em face dos conflitos apresentados, a adequação de técnicas de mediação à Bioética torna-se meio poderoso para concretização da resolução de dilemas que envolvem valores morais.

Deste cenário, nasce a Bioética Clínica, que busca encontrar condutas desejáveis no âmbito da relação de médico-paciente, possibilitando a máxima preservação dos valores pessoais e individuais dos envolvidos, afastando-se cada vez mais da medicina paternalista (CARREIRO, 2011).

Tomar decisões diante de um dilema moral na prática clínica torna-se, assim, uma necessidade e ao mesmo tempo traz consigo uma enorme dificuldade para os profissionais de saúde (MOTTA et al, 2016).

Assim, a tarefa de ponderar e mediar valores e dilemas morais não é fácil, necessitando de instrumentos capazes de solucionar os problemas éticos advindos especialmente num contexto hospitalar e que possa dirimir ou mitigar as vulnerabilidades. A deliberação em bioética é uma dessas ferramentas capazes de tornar a prática da medicina mais humana e menos defensiva.

Equipes multidisciplinares, deliberando em uma participação coordenada, ativa e coletiva, para auxiliar a tomada de decisões em dilemas morais, impulsionam para a nova experiência profissional, voltada ao melhoramento ético das práticas em saúde, assim como contribui para o exercício de uma medicina mais humana e afetuosa (GOMES, APARISI, 2017).

Metodologias de mediação de conflitos nesta área, como o de Albert Jonsen, professor emérito de ética médica na Faculdade de Medicina de Washington, baseiam-se nas indicações

Revista RBBA $\mid$ Revista Binacional Brasil Argentina Vol. 10, num. 2, dez/2021, p. 40-52 
médicas, preferências dos pacientes, qualidade de vida e aspectos contextuais, sendo que todos esses aspectos são relacionados de forma ordenada entre si, permitindo um raciocínio que pode ser compartilhado em linguagem comum e acessível por diversas áreas de conhecimento (URBAN, 2003).

Atualmente, um dos métodos mais utilizados na mediação de dilemas de valores é aquele desenvolvido por Diego Gracia, que descreve a deliberação como um processo de identificação de valores e deveres surgidos em torno de um caso concreto, visando a busca por uma solução ótima e, quando não for possível, ao menos aquelas menos prejudiciais ou mais prudentes aos envolvidos (NORA et al, 2015).

Ou seja, de acordo com o entendimento dos autores, deliberação coletiva pode auxiliar o próprio profissional de saúde a reconhecer e perceber os valores conflitantes, encontrar soluções possíveis e prudentes, principalmente com o reconhecimento da existência de um problema ético, que só se pode ser resolvido diante da maturação de decisões e cursos de ação aceitáveis.

Referido método parte da apresentação do caso clínico pela pessoa responsável pela tomada de decisão, com a subsequente discussão dos aspectos médicos e sociais envolvidos, oportunidade em que se mostra possível a identificação dos problemas e conflitos de valores morais existentes. Então, há a eleição daquele conflito de maior importância ou daquele que mais preocupa o responsável, a fim de que sejam percebidos cursos extremos e intermediários, dentre os quais será eleito o mais prudente, ou seja, o que atende ao maior número de valores detectados no caso concreto. A decisão final ainda é submetida a uma prova de consistência, que envolve a temporalidade, a publicidade e a legalidade, facilitando e trazendo maior segurança aos agentes envolvidos na questão, uma vez que são considerados aspectos circunstanciais, possibilidades existentes, disponibilidade e viabilidade de recursos, além dos interesses de todos os participantes da relação (ZOBOLI, 2012).

Abordagens baseadas em extremos de conduta são limitadas e, na maioria das vezes, baseiam-se em conclusões pouco racionalizadas, que deixam de considerar aspectos humanos importantes de uma relação médico-paciente, confluindo para uma acentuação do conflito preexistente.

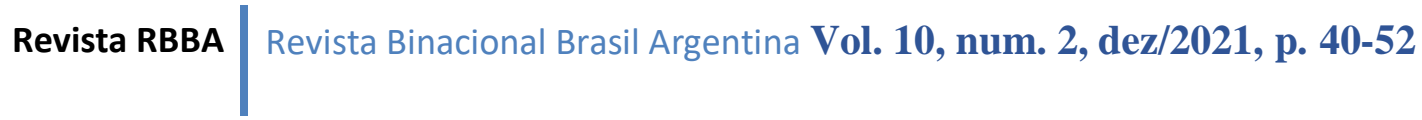




\section{CONSIDERAÇÕES FINAIS}

Diante da presente revisão bibliográfica, constata-se que técnicas deliberativas ou mediativas são úteis ao equacionamento de disputas, apresentando-se como alternativa disponível para todos aqueles que, em sua rotina diária enfrentam dilemas de valores morais, em especial nos ambientes de urgência hospitalar, cujas principais características são a vulnerabilidade emocional dos envolvidos e a necessária rapidez para tomadas de decisões difíceis (CARREIRO, 2011).

A ideia de conflito, portanto, diz respeito a elemento da própria vivência humana, permeando as relações médico-pacientes, especialmente diante da assimetria existente neste binômio.

O cuidado com a dor e o sofrimento humano figura no grande desafio a ser trabalhado pela área da saúde e, assim, na busca de um olhar mais aprofundado, modelos bioéticos são desenvolvidos como forma de perceber cada indivíduo na sua unidade, constituída de corpo e alma. O avanço tecnológico, ainda, dificulta ainda mais o relacionamento entre médico e paciente, porquanto reduz a possibilidade de escuta e diálogo, repercutindo na medicalização da vida e da morte, além da enorme imprecisão existente entre os limites éticos (PONTES et al, 2007).

Diante desse entendimento, profissionais da saúde podem constantemente se deparar com situações limítrofes, em que seus próprios valores entram em choque com os dos pacientes, situações muito comuns na prática clínica-hospitalar.

Fica, portanto, cada vez mais claro que a Bioética Principialista não é suficiente para dar conta das situações vividas no contexto médico-hospitalar, notadamente na prática clínica, de modo que a Bioética Clínica e seus métodos deliberativos se tornam ferramentas essenciais para auxiliar os profissionais de saúde em suas tomadas de decisões.

Mediar ou deliberar conflitos morais deve fazer parte da reeducação humana, proporcionando transformações tanto na prática profissional como na conduta da sociedade, além de permitir a própria confrontação com problemas éticos surgidos.

O exercício da medicina necessita de olhar empático, o que significa comunicar emoções, reconhecer sentimentos contraditórios, mas conjuntos e concomitantes. Não é ser capaz de se colocar no lugar do outro, mas sim de se conectar com o outro, reconhecer emoções e verdades conviventes.

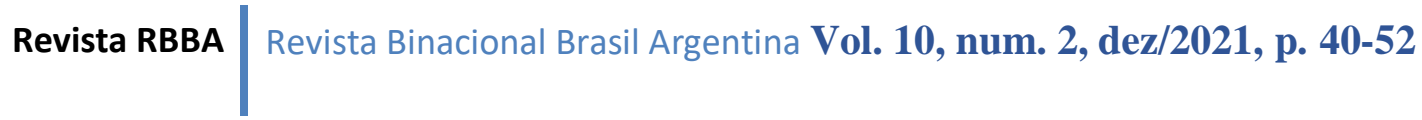


Em uma relação humana, desenvolver sentimentos em conjunto com o ser mais vulnerável é o mais puro estado de presença qualificada como afeto.

Quer parecer que, cada vez mais, profissionais da saúde, além da capacidade técnica, dependem do desenvolvimento de outras competências, relacionadas à ética e aos valores humanos, sobretudo ao desenvolvimento da compaixão e do respeito às diferenças entre os indivíduos.

Por outra via, profundas mudanças na sociedade contribuíram ainda mais para o surgimento de conflitos, porquanto pacientes revelam-se cada vez mais conscientes de seus direitos e passam a exigir profundas mudanças no comportamento médico, valendo-se de seus direitos, clamando pela autonomia e pela tomada de decisões de modo partilhado.

Com efeito, não é razoável imaginar que as deliberações e mediações bioéticas sejam as soluções precisas e definitivas para os dilemas que se apresentam, mas, sem dúvida, essas metodologias são capazes de aproximar os membros de uma equipe médica, pacientes e familiares, a partir da informação, da escuta ativa, do diálogo compassivo e da adoção de caminhos intermediários à melhor alternativa para um dado momento, ou seja, aquela que atende ao maior número de valores envolvidos em cada caso concreto.

O desenvolvimento dessas habilidades representa um olhar voltado para o paciente como ser humano único e individual, não mais apenas sobre a sua doença.

O desenvolvimento dessas habilidades representa a arte médica sendo humanizada. Uma medicina dos valores e afetos.

\section{REFERÊNCIAS}

BEAUCHAMP, Tom L. e CHILDRESS, James F. Princípios da Ética Biomédica. São Paulo: Edições Loyola, 2002.

BRASIL. CONSELHO FEDERAL DE MEDICINA (CFM - Brasil). Resolução Normativa n. ${ }^{\circ}$ 01/2016. Brasília, 2016.

CARREIRO, NMS. Mediação Bioética: busca de soluções compartilhadas para resolução de conflitos bioéticos (tese). Brasília: Universidade de Brasília; 2011.

FIGUEIREDO, Antonio Macena. Bioética Clínica e sua prática. In: Rev. Bioética, Brasília: 2011. Acessível em: https://revistabioetica.cfm.org.br/index.php/revista_bioetica/article/view/632

GOMES, Doris e APARISI, Juan Carlos Siurana. Deliberação Coletiva: uma contribuição contemporânea da bioética brasileira pra as práticas do SUS. In: Trab.Educ.Saúde, Rio de Janeiro, v. 15, n. 2, 2017.

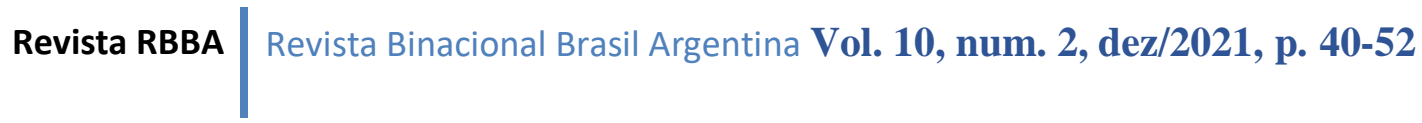


MEIRA, Affonso Renato. Bioética e vulnerabilidade: o médico e o paciente. Revista da Associação Médica Brasileira, v. 50, 2004. p. 249-250. Acessível em: https://www.scielo.br/j/ramb/a/58Gv6M9Qt5gZdyZ9m3yQkcM/?lang=pt\&format=pdf

MOTTA, Luís Claudio de Souza, OLIVEIRA, Lucas Nicolau; SILVA, Eugenio e SIQUEIRABATISTA, Rodrigo. Tomada de decisão em (bio)ética clínica: abordagens contemporâneas. In: Rev. Bioética, Brasília: 2016.

NASCIMENTO JÚNIOR, Pierre G.; MACÊDO GUIMARÃES, Teresinha Maria. A relação médico-paciente e seus aspectos psicodinâmicos. Revista Bioética, v. 11, n. 1, 2009. Acessível em: https://revistabioetica.cfm.org.br/index.php/revista_bioetica/article/view/152/156

NORA, Carlise Rigon Dalla et al. Deliberação ética em saúde: revisão integrativa da literatura. Revista Bioética, v. 23, n. 1, 2015. Acessível em: https://revistabioetica.cfm.org.br/index.php/revista_bioetica/article/view/992

OLIVEIRA, Aline Albuquerque S. Bioética e Direitos Humanos. São Paulo: Edições Loyola, 2011.

PAES, Bianca e ESCH, Georgia Lemos Abdalla. Comunicação e humanização na área da saúde. In: Comunicação, bioética e os últimos momentos, volume 1/organização Luís Fernando Rodrigues, Rodrigo Ales dos Santos; [colaboração Alejandro Enrique Barba Rodas... [et al.]] 1. Ed. - Rio de Janeiro: Rubio, 2021.

PAZINATTO, Márcia Maria. A relação médico-paciente na perspectiva da Recomendação CFM 1/2016. In: Rev. Bioética, Brasília: 2019.

PESSINI, Leo e BARCHIFONTAINE, Christian de P. Problemas Atuais de Bioética. $7^{\text {a }}$ Edição. São Paulo: Edições Loyola, 2005.

PONTES, Angela Cristina et al. Bioética e profissionais de saúde: algumas reflexões. Bioethikos [Internet], v. 1, n. 1, p. 68-75, 2007.

ROCHA, Bruno V. et al. Relação médico-paciente. Revista do médico residente, v. 13, n. 2, 2011. Acessível em: http://crmpr.org.br/publicacoes/cientificas/index.php/revista-do-medicoresidente/article/view/8/13

SOUZA, Virgínio Cândido Tosta, PESSINI, Leo e HOSSNE, William Saad. Bioética, religião, espiritualidade e a arte do cuidar na relação médico-paciente. In: Revista Bioethikos. Centro Universitário São Camilo: São Paulo, 2012. p. 181-190. Acessível em: http://www.saocamilosp.br/pdf/bioethikos/94/a7.pdf

URBAN, Cícero de Andrade. A bioética e a prática médica. In: Simpósio de Medicina e Direito. J.Vasc Br: 2003, vol. 02, n. ${ }^{\circ} 03$.

VASCONCELOS, Camila. Direito Médico e Bioética: história e judicialização da relação médico-paciente. Rio de Janeiro: Lumen Juris, 2020.

ZOBOLI, Elma. Bioética clínica na diversidade: a contribuição da proposta deliberativa de Diego Gracia. In: Revista BioEthikos. Centro Universitário São Camilo: São Paulo, 2012. Acessível

em: http://www.bioetica.org.br/library/modulos/varias_bioeticas/arquivos/Varias_Diversidade.pdf

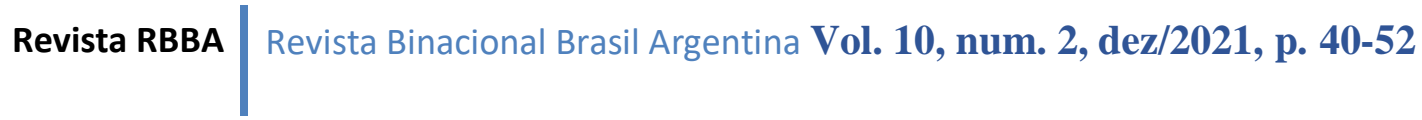

